\title{
CARACTERÍSTICAS FÍSICO-QUÍMICAS E SENSORIAIS DE TRÊS MARCAS DE LEITE DE VACA PASTEURIZADO E COMERCIALIZADO NA CIDADE DE ALFENAS-MG
}

Ana Rita Thomazela MACHADO ${ }^{1}$

João Eduardo de Carvalho CAMPOS ${ }^{2}$

Sílvia Silveira CLARETO ${ }^{3}$

Ana Lúcia Leite MORAES ${ }^{4}$

\author{
${ }^{1}$ Discente da Faculdade de Ciências Farmacêuticas da Unifal - MG; email: anaritatmachado@ @otmail.com \\ ${ }^{2}$ Discente da Faculdade de Ciências Farmacêuticas da Unifal - MG; email: eduardocampos88@outlook.com \\ ${ }^{3}$ Engenheira Química, professora da Universidade Federal de Alfenas; email:silvia.clareto@gmail.com \\ ${ }^{4}$ Farmacêutica, professora da Universidade Federal de Alfenas.;email: aleitemoraes@ gmail.com
}

\section{Recebido em: 30/05/2014 - Aprovado em: 15/09/2014 - Disponibilizado em: 15/12/2014}

\begin{abstract}
RESUMO: O leite de vaca é considerado um dos alimentos mais completos, devido ao seu alto teor de proteínas e sais minerais, além de ser importante fonte de cálcio. As maiores preocupações quanto à qualidade físico-química do leite estão relacionadas à remoção de substâncias químicas próprias ou à adição de substâncias estranhas a sua composição e à eficiência do seu tratamento térmico. Com o objetivo de avaliar as qualidades físico-químicas e sensoriais do leite pasteurizado comercializado em Alfenas-MG, amostras de três marcas diferentes de leite foram submetidas à avaliação físico-química e sensorial. Os dados obtidos foram submetidos à análise de variância e teste de Tukey $(\mathrm{p}<0,05)$. Na avaliação físico-química, as marcas 1 e 3 apresentaram-se dentro dos padrões estabelecidos pela Instrução normativa $\mathrm{n}^{\circ} 51$ enquanto que a marca 2 apresentou acidez titulável e densidade a $15^{\circ} \mathrm{C}$, incompatíveis com a legislação. $\mathrm{Na}$ análise sensorial, a marca 2 recebeu as piores notas, com valores do sabor e aroma extremamente baixos. Concluiu-se que das três marcas comercializadas na cidade de Alfenas-MG, duas possuem qualidades físico-químicas e sensoriais dentro dos padrões estabelecidos pela legislação e que a rejeição sensorial da marca 2 é decorrente das alterações verificadas nas análises físico-químicas.
\end{abstract}

Palavras-chave: Leite de vaca. Leite pasteurizado. Análise físico-química. Análise sensorial. Instrução normativa nº51.

\begin{abstract}
Cow milk is considered one of the most complete feed due to it's high protein value, minerals and for being an important source of calcium. The major concerns about physic-chemical quality are related to the removal of own chemichals in it's composition or adition of foreign substances and the efficiency of heat treatment. Aiming to evaluate the physico-chemical and sensory quality of pasteurized milk sold in Alfenas - MG, samples of three different brands of milk were subjected to physicochemical and sensory evaluation. The collected data were subjected to analysis of variance and Tukey test $(\mathrm{p}<0,05)$. In physico-chemical assessment, the brands 1 and 3 were within the standards set by IN $\mathrm{N}^{\circ} 51$ while the brand 2 showed titratable acidity and density at $15^{\circ} \mathrm{C}$ incompatible with the law. On sensory analysis, the brand 2 received the worst grades, with extremely low values of taste and aroma. Therefore, two of three milk brands sold in Alfenas-MG had physic-chemical and sensorial quality according to the legislation standards and the sensorial rejection of the brand 2 calls upon the changes found in the physico-chemical analysis.
\end{abstract}

Key words: Cow milk. Pasteurized milk. Physico-chemical analysis. Sensory analysis. Regulatory Instruction 51.

\section{INTRODUÇÃO}

O leite é definido como o produto oriundo da ordenha completa e ininterrupta, em condições de higiene, de vacas sadias, bem alimentadas e descansadas (BRASIL,
2002). É considerado um dos alimentos mais completos, do ponto de vista nutricional, devido ao seu alto teor de proteínas e sais minerais, além de ser importante fonte de cálcio. (TAMANINI et al., 2007; LUZ et al., 
2011). Esta composição pode ser influenciada por diversos fatores, tais como a genética bovina, fatores fisiológicos e alimentação, condições da lactação e exercícios. Estes fatores também podem estar correlacionados com outros parâmetros, como a região de produção ou estação do ano (GAUCHER et al., 2008).

O leite bovino é um fluido composto por uma série de nutrientes sintetizados nas glândulas mamárias, a partir de precursores derivados da alimentação e metabolismo. Os componentes incluem água, glicídeos (basicamente lactose), gordura, proteína (principalmente caseína e albumina), minerais e vitaminas. Sendo secretado como uma mistura de componentes individuais (GONZÁLES, 2001).

A pasteurização do leite tem como objetivo principal eliminar os microorganismos patogênicos que possam contaminar o leite, tornando-o um produto inócuo ao consumo humano. A legislação brasileira estabelece que a pasteurização deve ser realizada submetendo-se o leite à temperatura entre $72^{\circ} \mathrm{C}$ a $75^{\circ} \mathrm{C}$ por 15 a 20 segundos (BRASIL, 1996a). O tratamento térmico pode provocar mudanças nas propriedades físico-químicas do leite, mas estas normalmente são insignificantes quando a temperatura não ultrapassa $60^{\circ} \mathrm{C}$ (SOUZA et al., 2013).
A necessidade de melhoria da qualidade do leite no Brasil resultou em medidas oficializadas pela Instrução Normativa $\mathrm{n}^{\mathrm{o}} 51$ (IN $\mathrm{n}^{\mathrm{o}} 51$ ), aprovada em setembro de 2002. Esta norma é composta por regulamentos que tratam da produção, identidade, qualidade, coleta e transporte de leite, visando a produção de leite sadio, seguro e confiável ao consumidor. $\mathrm{O}$ objetivo da $\mathrm{IN} \mathrm{n}^{\circ} 51$ é fixar os requisitos mínimos que devem ser observados na qualidade do leite cru, do leite cru refrigerado e do leite pasteurizado, enquanto perdurar a produção desse tipo de leite destinado ao comércio nacional (LUZ et al., 2011).

A análise sensorial possibilita diferenciar e caracterizar o produto pelos atributos sensoriais, determinando se o produto é aceitável ou não pelo consumidor. No setor de alimentos, a manutenção das características sensoriais do produto contribui para a lealdade do consumidor em um mercado cada vez mais competitivo, sendo parte inerente ao plano de controle de qualidade de uma indústria (TEIXEIRA, 2009).

Neste contexto, o presente estudo tem como objetivo a análise físico-química e sensorial de três marcas de leite bovino pasteurizado comercializados na cidade de Alfenas, Minas Gerais. 


\section{MATERIAL E MÉTODOS}

O leite de vaca pasteurizado foi obtido em um supermercado localizado no município de Alfenas-MG. As amostras foram designadas de 1,2 e 3 referente às três marcas analisadas.

Recolheu-se neste estabelecimento, aleatoriamente, $10 \%$ do número de embalagens de mesmo lote, de cada marca a ser analisada. As amostras foram transportadas em caixas isotérmicas até o laboratório, onde foram realizadas as análises.

No Laboratório de Bromatologia da Universidade Federal de Alfenas (UnifalMG), foram realizadas as seguintes análises: densidade a $15^{\circ} \mathrm{C}$, acidez em graus Dornic, extrato seco total (EST), extrato seco desengordurado (ESD), lipídeos e cinzas de acordo com o IAL (2008) e pH, açúcares redutores (lactose) e proteínas de acordo com a AOAC (2005).

A análise sensorial foi realizada no Laboratório de Análise Sensorial da Universidade Federal de Alfenas (UnifalMG). A seleção dos 74 avaliadores, de ambos os sexos, foi de forma aleatória e voluntária. Foram oferecidos cerca de $40 \mathrm{~mL}$ de leite de vaca pasteurizado, resfriado a $12^{\circ} \mathrm{C}$, em copos descartáveis codificados com números de três dígitos em ordem casualizada e um copo com água à temperatura ambiente, para enxaguar a boca antes de cada julgamento. As avaliações foram conduzidas em cabines individuais. Os provadores avaliaram o grau de aceitação e aparência do leite por meio de uma escala hedônica de nove pontos, onde nove corresponde à "gostei extremamente", cinco corresponde à "indiferente" e um corresponde à "desgostei extremamente" (MEILGAARD, 1999).

As análises físico-químicas foram realizadas em triplicata. Os resultados foram submetidos à análise de variância e as médias analisadas pelo teste de Tukey ao nível de 5\% de significância. Para interpretação da análise sensorial também foi utilizado análise de variância e teste de Tukey ao nível de 5\% de significância.

Os dados foram analisados com o uso do software estatístico SAS (SAS/INSTITUTE, 1997).

\section{RESULTADOS E DISCUSSÃO}

No Brasil, a Instrução Normativa 51 do MAPA (BRASIL, 2002) regulamenta os requisitos mínimos que devem ser observados na identidade e na qualidade do leite cru, do leite refrigerado e do leite pasteurizado. São estabelecidos como padrões mínimos: 3\% de gordura (padronizado), acidez de 0,14 a 0,18\% de ácido lático (14 a $\left.18^{\circ} \mathrm{D}\right), 8,4 \%$ de sólidos não gordurosos (ESD), 2,9\% de proteína total e densidade relativa a $15^{\circ} \mathrm{C}$ de 1,028 a $1,034 \mathrm{~g} / \mathrm{mL}$.

De acordo com a tabela 01 verificouse que as marcas 1 e 3 apresentaram 
características físico-químicas dentro dos padrões estabelecidos pela instrução normativa 51, enquanto a marca 2 apresentou acidez titulável acima (37,50 ${ }^{\circ}$ Dornic) e densidade a $15^{\circ} \mathrm{C}$ abaixo $(1,027 \mathrm{~g} / \mathrm{mL})$ da estabelecida pela legislação e pH mais ácido $(5,86)$ que as demais.

Valores de acidez titulável acima de $24^{\circ} \mathrm{D}$, acompanhados de valores de $\mathrm{pH}$ próximos à 6,1 , indicam que o leite não passou por uma correta pasteurização a $72^{\circ} \mathrm{C}$
(EMBRAPA, 2014). Fonseca et al. (2010) analisaram 30 amostras de leite pasteurizado tipo C comercializados na cidade de JanaúbaMG e encontraram um valor médio de acidez titulável de $17^{\circ}$ Dornic. A implicação usual de altos valores de acidez no leite é que este contém elevados níveis de ácido lático. A causa mais provável é a fermentação da lactose por bactérias (GONZÁLES, DURR, FONTANELI, 2001).

Tabela 01 - Características físico-químicas de três marcas de leite de vaca pasteurizado.

\begin{tabular}{cccc}
\hline Análises físico-químicas & \multicolumn{3}{c}{ Leite } \\
\cline { 2 - 4 } & Marca 1 & Marca 2 & Marca 3 \\
\hline Acidez titulável ( ${ }^{\circ}$ Dornic) & $14,50^{\mathrm{b}} \pm 0,00$ & $37,50^{\mathrm{a}} \pm 0,00$ & $14,50^{\mathrm{b}} \pm 0,00$ \\
Cinzas (\%) & $2,81^{\mathrm{ns}} \pm 0,01$ & $2,66^{\mathrm{ns}} \pm 0,04$ & $2,76^{\mathrm{ns}} \pm 0,13$ \\
Densidade a $15^{\circ} \mathrm{C}(\mathrm{g} / \mathrm{mL})$ & $1,028^{\mathrm{b}} \pm 0,00$ & $1,027^{\mathrm{a}} \pm 0,00$ & $1,028^{\mathrm{b}} \pm 0,00$ \\
pH & $6,61^{\mathrm{a}} \pm 0,01$ & $5,86^{\mathrm{b}} \pm 0,00$ & $6,60^{\mathrm{a}} \pm 0,01$ \\
Extrato seco desengordurado & $8,98 \pm 0,24$ & $8,78 \pm 0,29$ & $9,15 \pm 0,20$ \\
- ESD (\%) & & & \\
Extrato seco total - EST $(\%)$ & $12,33^{\mathrm{a}, \mathrm{b}} \pm 0,30$ & $11,93^{\mathrm{b}} \pm 0,35$ & $12,60^{\mathrm{a}} \pm 0,30$ \\
Teor de lipídeos $(\%)$ & $3,35^{\mathrm{a}} \pm 0,06$ & $3,15^{\mathrm{b}} \pm 0,06$ & $3,45^{\mathrm{a} \pm 0,10}$ \\
Teor de proteínas $(\%)$ & $3,34^{\mathrm{a}} \pm 0,07$ & $2,95^{\mathrm{b}} \pm 0,09$ & $3,40^{\mathrm{a}} \pm 0,05$ \\
Umidade $(\%)$ & $87,67^{\mathrm{a}, \mathrm{b}} \pm 0,30$ & $88,07^{\mathrm{a}} \pm 0,35$ & $87,40^{\mathrm{b}} \pm 0,30$ \\
\hline
\end{tabular}

Médias acompanhadas de letras diferentes, na mesma linha diferem entre si significativamente $(\mathrm{p}<0,05)$. ns:não significativo.*fator de correção utilizado: 6,38. Fonte: o autor.

A determinação da densidade do leite é considerada uma análise de rotina importante, pois valores destoantes da legislação podem indicar fraude por adição de água ou por retirada da nata ou adição de qualquer outro corretivo (PAIVA, 2007). A densidade do leite é uma relação entre seu peso e volume.

Sena et al., (2001) encontraram resultado médio de $1,027 \mathrm{~g} / \mathrm{mL}$ em 71 amostras de leite pasteurizado tipo $\mathrm{C}$, valor também considerado fora dos padrões.

Silva et al. (2008) verificaram que $1,4 \%$ das amostras de leite analisadas no estado de Alagoas apresentaram-se fora dos padrões físico-químicos, o que indicou fraude por adição de água quando os valores obtidos foram abaixo da densidade adequada.

Segundo Fonseca et al. (2008), o extrato seco desengordurado, assim como o teor de gorduras e proteínas, podem sofrer 
influências regionais associadas às flutuações estacionais. Geralmente os teores de sólidos totais e extrato seco desengordurado alcançam maiores valores no outono e menores na primavera.

Os valores de gorduras e proteínas encontrados nas três marcas de leite pasteurizados analisadas, estão dentro dos padrões normativos. Dados semelhantes foram encontrados por Ribas et al. (2004), com 3,24\% de proteínas e 3,69\% de gorduras. Sgarbieri (2004) também detectou valores próximos: $3,3 \%$ de proteínas e 3,8\% de gordura. Torres et al. encontraram $3,24 \%$ de proteínas e $3,1 \%$ de gordura em leite bovino pasteurizado tipo $\mathrm{C}$.

O conteúdo de sólidos totais de uma amostra de leite expressa a concentração de proteínas, lipídeos, carboidratos e minerais. Quanto mais elevada for a quantidade de sólidos totais apresentada por uma amostra de leite, melhor será o seu rendimento para a indústria de laticínios (QUEIROGA et al., 2010). Na análise de EST, a porcentagem encontrada nas três marcas variou de 11,93 a 12,60\%. Queiroga et al. (2010), ao analisar 48 amostras de leite pasteurizado na Paraíba, obtiveram resultados médios menores (11,31\%) e Ribas et al. (2004) encontraram uma porcentagem de $12,32 \%$ de EST nas amostras de leite.

As características físico-químicas do leite podem ser alteradas devido às condições genéticas, nutricionais e ambientais das vacas, aos processos de obtenção, armazenamento, transporte e beneficiamento do leite, e às fraudes. Portanto, corroborando os valores de acidez titulável e de densidade a $15^{\circ} \mathrm{C}$ do presente estudo com os valores estipulados pela legislação, pode-se verificar que o leite pasteurizado da marca 2 não seguiu os procedimentos estabelecidos pelas boas práticas de fabricação e, possivelmente, sofreu adulterações.

É de suma importância avaliar as características físico-químicas do leite, a fim de considerar a possibilidade de ocorrência de fraudes econômicas, estabelecer base para pagamento e verificar o seu estado de conservação (AGENESE, et al., 2002).

Tabela 02 - Características sensoriais de três marcas de leite de vaca pasteurizado.

\begin{tabular}{cccc}
\hline Análise & \multicolumn{3}{c}{ Leite } \\
\cline { 2 - 4 } sensorial & Marca 1 & Marca 2 & Marca 3 \\
\hline Aroma & $6,69^{\mathrm{a}}$ & $5,81^{\mathrm{b}}$ & $6,56^{\mathrm{a}}$ \\
Sabor & $6,53^{\mathrm{a}}$ & $3,69^{\mathrm{b}}$ & $6,04^{\mathrm{a}}$ \\
Textura & $6,86^{\mathrm{a}}$ & $5,85^{\mathrm{b}}$ & $6,83^{\mathrm{a}}$ \\
Aparência & $7,55^{\mathrm{a}}$ & $6,88^{\mathrm{b}}$ & $7,25^{\mathrm{a}, \mathrm{b}}$ \\
Impressão & $6,96^{\mathrm{a}}$ & $6,05^{\mathrm{a}}$ & $6,48^{\mathrm{a}}$ \\
\hline
\end{tabular}

Médias acompanhadas de letras diferentes, na mesma linha diferem entre si significativamente $(\mathrm{p}<0,05)$. Fonte: o autor

De acordo com a tabela 02, pode-se observar que a marca 2 obteve as piores notas dentre as amostras analisadas. Destaca-se as notas 3,69 (desgostei moderadamente) para o sabor e 5,81 (nem gostei/nem desgostei) para o aroma, que podem ser relacionados com a 
alta acidez percebida pelo paladar e pelo olfato dos provadores. As marcas 1 e 3 obtiveram coeficientes de avaliação que indicam um leite mais atrativo para 0 consumidor.

Homsey (2000) descreve o leite como sendo uma bebida doce, devido à lactose, salgada, devido aos sais inorgânicos e cremosa devido à gordura. Fatores como o estado sanitário do rebanho, alimentação e manejo dos animais e equipamentos durante a ordenha, presença de microrganismos, e cuidados posteriores até o momento do consumo final influenciam no sabor do leite (BODYFELT et al., 1988).

\section{CONCLUSÃO}

Conclui-se que das três marcas de leite bovino pasteurizado comercializados em Alfenas-MG, as marcas 1 e 3 apresentaram resultados físico-químicos e sensoriais dentro dos padrões exigidos pela legislação em vigor, mas a marca 2 apresentou uma acidez elevada que colaborou para a sua rejeição sensorial. É necessário um monitoramento contínuo para garantir a qualidade do produto e uma ingestão alimentar satisfatória.

\section{REFERÊNCIAS BIBLIOGRÁFICAS}

AOAC - ASSOCIATION OF OFFICIAL ANALYTICAL CHEMISTRY. Official methods of analysis of the AOAC: supplement. Food composition additives;
Natural Contaminants. 16 ed. Gaithersburg: AOAC, 2005.

BODYFELT, F. W.; TOBIAS, J.; TROUT, G. M. The Sensory Evaluation of Dairy Products. Nova York: Publication New York, AVI, Van Nostrand Reinhold, 1988. 598p

BRASIL. Ministério da Agricultura, Pecuária e Abastecimento. Departamento de inspeção de produtos de origem animal. Instrução Normativa $n^{\circ} 51$. Aprova e oficializa o Regulamento Técnico de identidade e qualidade de leite pasteurizado tipo $\mathbf{C}$ refrigerado. Diário Oficial da União. Brasília, 2002.

EMBRAPA. Agência de Informação Embrapa. Acidez Titulável. 2007. Disponível em:

<http://www.agencia.cnptia.embrapa.br/Agen cia8/AG01/arvore/AG01_194_21720039246. html>. Acesso em: 20 mai. 2014.

GAUCHER, I.; BOUBELLOUTA, T.; BEAUCHER, E.; PIOT, M. Investigation of the effects of season, milking region, sterilisation process and storage conditions on milk and UHT milk physico-chemical characteristics: a multidimensional statistical approach. Dairy Sci, v. 88, p. 291.312, 2008.

GONZÁLEZ, F. H. D.; DÜRR, J. W.; FONTANELI, R. S. Uso do leite para monitorar a nutrição e o metabolismo de vacas leiteiras. Biblioteca Setorial da Faculdade de Medicina Veterinária da UFRGS, Porto Alegre, 2001.

HOMSEY, C. Discovering Dairy Flavors. Food Product Design, 2000. Disponível em: <http://www.foodproductdesign.com/ archive/2000/1200ap>. Acesso em: 20 de maio de 2014

INSTITUTO ADOLFO LUTZ. Normas analíticas: métodos químicos e físicos para análise de alimentos. São Paulo: Instituto Adolfo Lutz, 2008. 1020p. Disponível em: 
<http://www.ial.sp.gov.br> Acesso em: 20 ago. 2013.

LUZ, D. F.; BICALHO, F. A.; OLIVEIRA, M. V. M.; SIMÕ̃ES, A. R. P. Avaliação microbiológica em leite pasteurizado e cru refrigerado de produtores da região do Alto Pantanal Sul-Mato-Grossense. Revista

Agrarian, Dourados, v. 4, n. 14, p. 367-374, 2011.

MEILGAARD, M.; CIVILLE, G. V.; CARR, B. T. Sensory Evaluation Techniques. 3 ed. Boca Ration, CRC Press, 1999.

PAIVA, R. M. B. Avaliação físico-química e microbiológica de leite pasteurizado tipo $\mathrm{C}$ distribuído em programa social governamental. 2007. 76f. Dissertação (Mestrado em Medicina Veterinária) Universidade Federal de Minas Gerais.

QUEIROGA, R. C. R. E.; CAMBUIM, R. B.; OLIVEIRA, M. E. G.; VIANNA, R. P. T.; SOUZA, E. L. Microbiological and physicochemistry quality of pasteurized type $\mathrm{C}$ milk distribuited by the "Milk of Paraíba" program. Nutrire: rev. Soc. Bras. Alim. Nutr. = J. Brazilian Soc. Food. Nutr., São Paulo, SP, v. 35, n. 1, p. 97-109, abr. 2010.

RIBAS, N. P.; HARTMANN, W.; MONARDES, H. G.; ANDRADE, U. V. C. Sólidos totais do leite em amostras de tanque nos estados do Paraná, Santa Catarina e São Paulo. Rev. Bras. Zootec., v. 33, n. 6, p. 2343-2350, 2004.

\section{SAS INSTITUTE. SAS/STAT software:} changes and enhancements through release 6.12. cary: Statistical Analysis System institute, 1997.

SCARBIERI, V. C. Propriedades fisiológicasfuncionais das proteínas do soro do leite. Rev. Nutr., v. 17, n.4, p. 397-409, 2004.

SENA, M. J.; MENDES, E. S.; ALMEIDA, C. C. et al. Qualidade físico-química e microbiológica do leite pasteurizado tipo $\mathrm{C}$ comercializado em Recife. Revista do Instituto de Laticínios Cândido Tostes, v. 56, n. 321, p. 241-248, 2001.

SOUZA, A. K.; FIORINI, J. E.; MORAES, A. L. L.; OLIVEIRA, N. M. S.; CLARETO, S. S.; NASCIMENTO, L. C. Características microbiológicas e físico-químicas do leite de cabra submetido à pasteurização e ao congelamento, comercializado na cidade de Alfenas-MG. Revista Higiene Alimentar, v. 17, n. 106, p. 100-104, 2003.

TAMANINI, R.; SILVA, L. C. da.; MONTEIRO, A. A.; MAGNANI, D. F.; BARROS, M. A.; BELOTI, V. Avaliação da qualidade microbiológica e dos parâmetros enzimáticos da pasteurização de leite tipo "C" produzido na região norte do Paraná. Semina: Ciências Agrárias, Londrina, v. 28, n. 3, p. 449-454, 2007.

TEIXEIRA, L.V. Análise Sensorial na indústria de alimentos. Rev. Inst. Latic. "Cândido Tostes", Jan/Fev, no 366, 64: 12-21, 2009.

TIMM, C. D.; GONZALEZ, H. L.; OLIVEIRA, D. S.; BÜCHLE, J.; ALEXIS, M. A.; COELHO, F. J. O.; PORTO, C. Avaliação da qualidade microbiológica do leite pasteurizado Integral produzido em microusinas da região sul do Rio Grande do Sul. Revista Higiene Alimentar, v. 17, n. 106, p. 100-104, 2003.

TORRES, E. A. F. S.; CAMPOS, N. C.; DUARTE, M.; GARBELOTTI, M. L.; PHILIPPI, S. T.; MINAZZI-RODRIGUES, R. S. Composição centesimal e valor calórico de alimentos de origem animal. Ciênc. Tecnol. Alim., v. 20, n. 2, p. 145-150, 2000. 